\title{
X-RAYS FROM SPACE
}

\author{
M. ARNAUD \\ Service d'Astrophysique, CEN Saclay, F-91191 Gif-sur-Yvette \\ Cedex, France
}

RESUME: Le rayonnement $X$ d'origine spatiale ne peut atteindre la surface de la Terre car il est absorbé par l'atmosphère. Aussi l'Astronomie X n'a t'elle pu se développer qu'avec l'avènement des techniques spatiales. On peut fixer sa naissance au 12 Juin 1962 (i1 y a donc 25 ans), jour de la première observation d'une source $X$ lointaine (Scorpius X-1). Tandis que les expériences pionnières (UHURU,...) révélè̀rent l'existence même de sources $\mathrm{X}$, les plus récentes (telles que les satellites EINSTEIN et EXOSAT ) mirent en oeuvre de véritables observatoires d'une haute polyvalence et d'une technologie avancée, qui associaient l'imagerie aux analyses spectroscopiques. Ces vols-fusées et ces satellites ont montré que des émissions de rayons $\mathrm{X}$ se produisaient dans la plupart des sites astrophysiques: étoiles, systèmes stellaires binaires, milieu interstellaire, galaxies actives et quasars, amas de galaxies, milieu intra-amas. Comme les rayons $\mathrm{X}$ sont émis lors de processus physiques mettant en jeu des énergies élevées (émission thermique de plasmas très chauds, émission synchrotron de particules relativistes,...) ils ouvrent une fenêtre sur les phénomènes cataclysmiques dans l'Univers tels que les explosions stellaires et la propagation subséquente d'ondes de choc ou, par exemple, l'accrétion par des trous noirs de quantités énormes de matière. L'Astronomie X n'a peut-être pas encore atteint sa maturité: on attend des progès spectaculaires de la nouvelle génération d'instruments, tels que les spectromètres à haute résolution, qui doit équiper le futur satellite européen XMM. L'interprétation de la moisson de données qu'ils devraient apporter nécessitera une collaboration toujours plus intense avec les spectroscopistes et les physiciens atomistes

\footnotetext{
ABSTRACT: X-rays from outer space are prevented by the atmosphere from reaching the ground.Therefore, $X$-rays astronomy developed as a new branch of astrophysics only with the advent of spatial technologies. It was born 25 years ago, on the 12 of June 1962, with the first observation of a remote X-ray source (Scorpius X-1). Whereas the pioneering experiments (UHURU,...) brought to light the very existence of $X$-ray sources, the most recent ones (such as the EINSTEIN and EXOSAT satellites) were versatile and sophisticated observatories which performed spectroscopic studies as well as imagery. These rocket and satellite experiments have revealed that X-rays are emitted in most astrophysical sites: stars, binary stellar systems, interstellar medium, active galaxies and quasars, galaxy clusters, intercluster medium. Since $\mathrm{X}$-rays originate in highly energetic processes (thermal emission of very hot plasmas, synchrotron emission of relativistic particles,...) they shed some light on cataclysmic phenomena such as stellar explosions and propagation of the resulting shock wave, huge matter infalls onto the surface of black holes,...X-ray astronomy may have not yet truly come of age: drastic improvements are expected from a new generation of instruments, such as the high resolution spectrometers which are to be implemented on board the future ESA satellite XMM. The interpretation of the wealth of data they will provide will require an ever increasing collaboration with specialists in spectroscopy and atomic processes.
} 


\section{A GENERAL OVERVIEW}

For several centuries astronomy has been confined to the observation of the visible light of stellar objects. The observation of the Universe in other spectral windows (radio, infrared, ultraviolet, $X$ and $\gamma$ rays) marked the eve of a new age of Astrophysics. $\mathrm{X}$ rays are emitted in very energetic physical processes and their detection may therefore bring us essential informations on violent phenomena in the Universe.

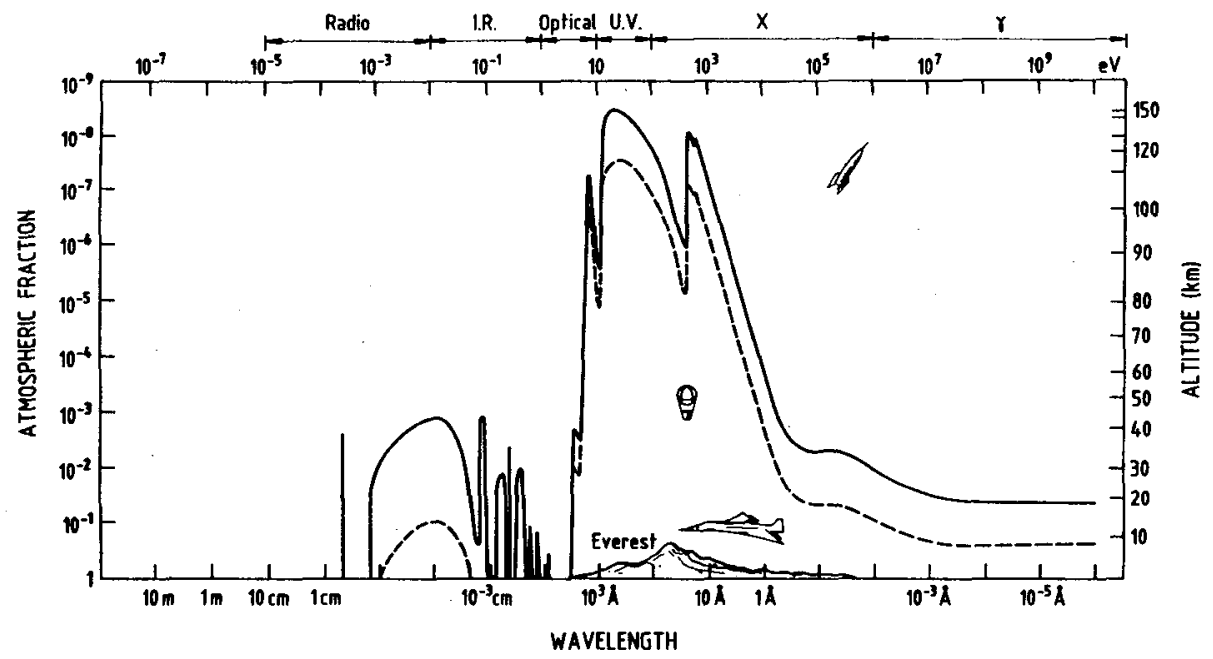

Figure 1 Maximum altitudes below which more than $50 \%$ (full) and $99 \%$ (dashed) of the incoming radiation is absorbed as a function of the wavelength.

Unfortunately $\mathrm{X}$ rays produced in space are absorbed by the atmosphere and cannot be detected from the ground (see figure 1). Therefore X-ray astronomy could develop as a new branch of astrophysics only with the advent of space technologies.

The first X-ray source to be discovered was far from exotic: indeed in 1948 a photographic emulsion aboard a rocket revealed that the sun was an X-ray source. It was far easier to detect this weak (one million less energy emitted in X-rays than in the visible spectrum) but nearby source than remote emitters. Indeed 14 years elapsed before the first observation of an X-ray source outside the solar system: the binary stellar system Scorpius X-1. 
Rocket experiments suffer from their limited flight time. Satellites, which are immune from this shortcoming, enabled the development of true spatial X-ray observatories. Another milestone was the introduction in 1978 of X-ray optics: the use of grazing incidence mirrors led to a drastic improvement in sensitivity (mirrors acting as collectors) and spatial resolution.

The most recent satellites (such as the U.S. satellite 'EINSTEIN' or the european one EXOSAT) were versatile observatories which performed spectroscopic studies as well as imagery (see figure 2). The progress made since the time of the pioneering rocket experiment is well illustrated by the following figure: the increase in sensitivity, a factor $10^{6}$, is the same as between the Mount Palomar 5m telescope and the telescope devised by Galileo in 1610 .

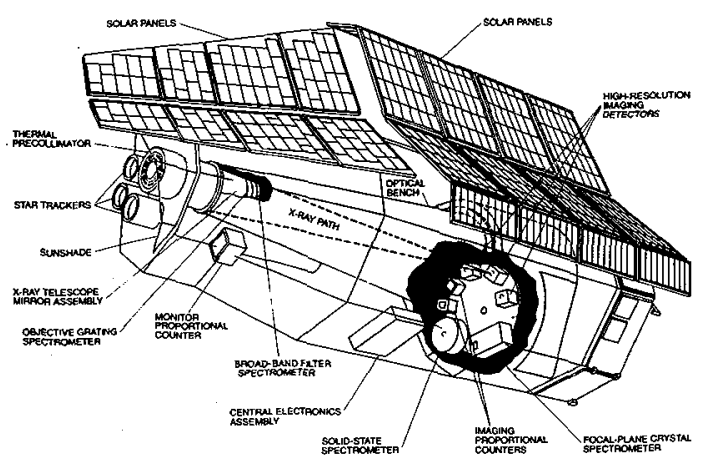

(a)

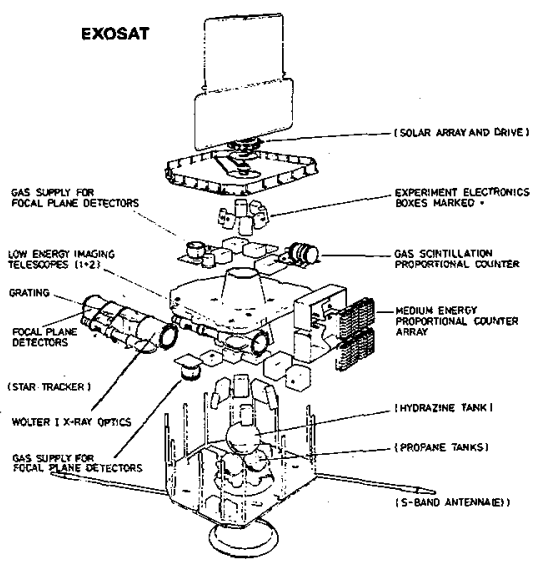

(b)

Figure 2 (a) The EINSTEIN observatory launched in 1978. A turn table at the focus of the telescope carries four different instruments: two spectrometers and two devices with imaging capability.

(b) The EXOSAT satellite launched in 1983. In addition to focal instruments devoted to imagery, it incorporated large area proportional counters. 
At the sight of such a spectacular advance one must not jump to the conclusion that $\mathrm{X}$-ray observation has now became a routine undertaking. Much progress is still to be done. The energy resolution falls short of reaching the value currently obtained in laboratory plasma experiments (see figures 3 and 7).
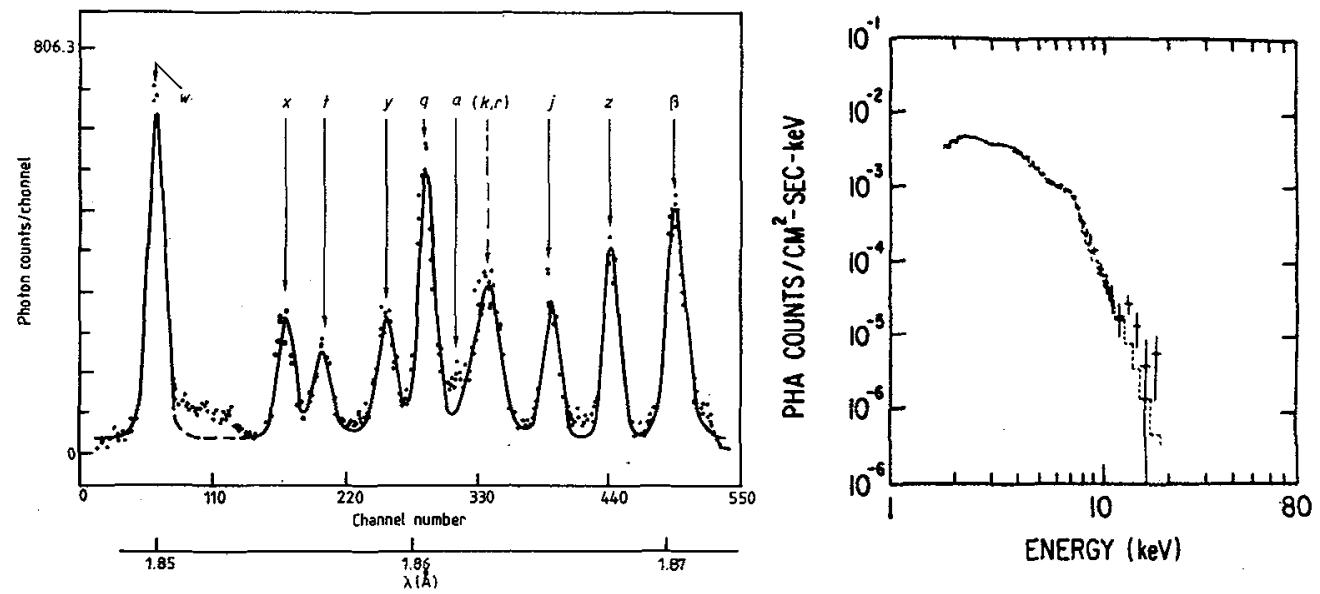

Figure 3 Observations of the Iron line complex around $6.7 \mathrm{keV}$ : (a) in the Princeton Laboratory Tokamak.

(b) in a galaxy cluster using a proportional counter.

Up to now most spectroscopic studies have relied on proportional counters, which measure the energy of each incoming photon. The introduction of solid state devices (SiLi diodes) have led to a sharp improvement in spectral resolution (160 ev currently achieved) with respect to the first generation gas counters $(\Delta \mathrm{E} / \mathrm{E} \approx 0.1$ at $6 \mathrm{keV})$. However high spatial and spectral resolution were up to now conflicting goals. A new generation of instruments (such as CCD devices, bolometers, curved Bragg spectrometers) aims at solving these problems. They will be presented in the last section.

Besides one of the major difficulty resides in the very use of satellites. Embarked experiments must be extremely reliable (as repair is impossible), must work in the hostile space environment and must be as light, small sized and power saving as possible.

In conclusion I would like to emphasize that X-ray observatories are costly objects, the development of which takes a long time (typically 10 to 15 years) and are usually (and more and more frequently) devised in the framework of international collaborations. 
These satellite and rocket experiments have provided a harvest of data on every class of astrophysical objects. Indeed, as the reader may know, the Universe displays a hierarchy of nested structures: galaxies, made of myriads of stars, are themselves gathered in clusters (typically 100 to 1000 galaxies). At still larger scale clusters may belong to superclusters. At each level of this hierarchy X-ray emission occurs.

- As the Sun, most stars are surrounded by a corona hot enough $\left(T=10^{6}\right.$ to $10^{7} \mathrm{~K}$ ) to produce X-rays. A much stronger emission is found in binary stellar systems which will be described in the next section.

- At the galactic level, the emission originates in the hot phase of the gaseous interstellar medium (see below) or in the bulge of the so-called active galaxies (100 to 100000 (US) billion times the solar luminosity). The strongest emission comes from the Quasars; the very term QUAsi-StellAR objects was coined by the radio astronomers who discovered them, on account of their point-like aspect. In this case, the observed luminosity is so enormous that the processes at work to supply the required energy still remain hypothetical: some explanations rely on huge matter infalls onto a supermassive black hole. As quasars are the most remote objects ever detected, the radiation we detect was emitted in a far away past (typically some $10^{9}$ years) and their study is thus of great interest to cosmologists.

- At the next level of the hierarchy X-ray astronomy revealed the existence of a hot gas between galaxies inside clusters. The existence of ionized Iron lines proved that this gas had been enriched through stellar nucleosynthesis. Its study may therefore be a clue to the understanding of cluster formation and evolution.

Besides it is an unique tool for the study of the dark matter. Indeed the cluster's total mass, as estimated by the requirement of gravitational boundness, far exceeds the mass till now directly observed This is the so called missing mass problem. The gas is in hydrostatical equilibrium in the gravitational well created by the cluster's total mass. and thus is a tracer of its distribution.

- To the general surprise the earliest rocket-borne experiments detected a diffuse X-ray emission coming from all over the sky. Its origin is still controversial. The presence of a hot gas spreading between galaxy clusters themselves might provide an explanation. An alternative proposal involves the summed contributions of unresolved remote sources (such as quasars).

In the following section, we shall illustrate on some representative examples the physical mechanisms involved in space X-ray emission. 


\section{TWO EXAMPLES OF X-RAY SOURCES: BINARY STELLAR SYSTEMS AND SUPERNOVAE REMNANTS}

\section{I - binary stellar systems}

Nearly $50 \%$ of stars in our galaxy belong to binary systems (associations of two stars in a bound system resulting from mutual gravitational attraction).

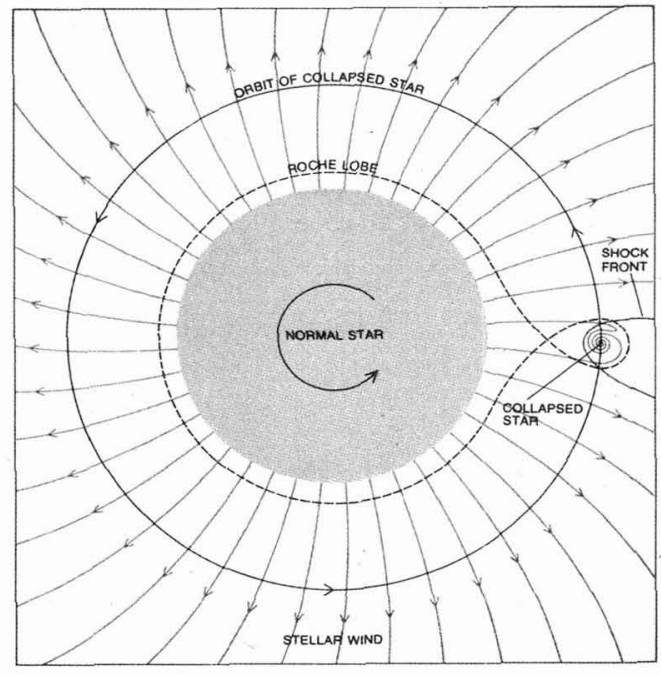

(a)

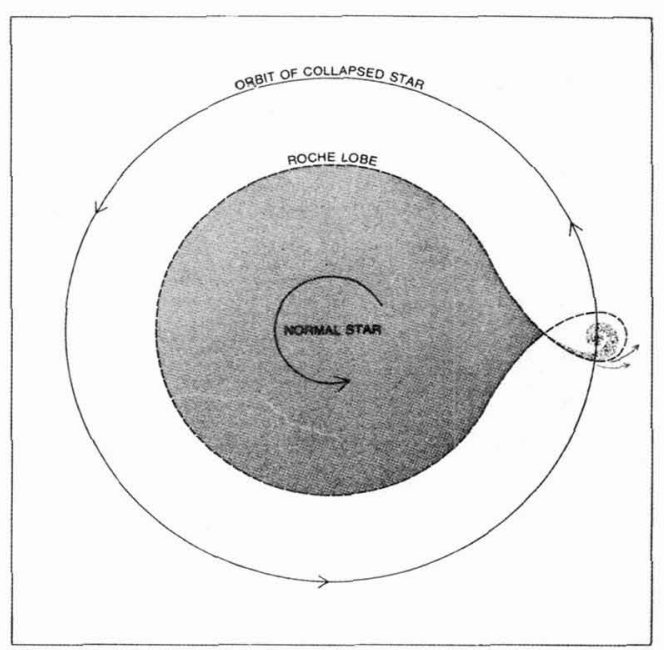

(b)

Figure 4: In a binary stellar system the normal star may loose matter for the benefit of the compact star either via a stellar wind (a) or via the direct attraction of the outer shells by its companion (b).

The generic system is constituted by a normal star and a compact companion, typically a neutron star (see figure 4). As the reader may know, neutron stars are created as a normal star explodes, ejecting its outer shells into space while its heart undergoes an incomplete gravitational collapse stopped by the degeneracy pressure of neutrons. In such systems matter is transferred from the normal star to its companion, a phenomenon known as accretion. $\mathrm{X}$-ray emission originates in the conversion of kinetic energy into heat when the infalling matter reaches the compact star surface. The gravitational field created by a neutron star (typically the same mass as the Sun within a $15 \mathrm{~km}$ radius) is so intense that the radiation energy produced in such a process amounts to almost $10 \%$ of the rest mass of the accreted matter. 
The mechanism of the infall depends on the nature of the binary system (masses of the stars, magnetic field):

- A first class roughly regroups low mass systems. In that case, matter at the surface of the normal star is more attracted by the compact star than by the very star it belongs to. Accretion then occurs trough the Lagrange point, the point were the two gravitational forces equilibrate (see figure $4 \mathrm{~b}$ ). The infalling matter builds up a rotating disk around the compact star. If this latter is only weakly magnetized, the inner rim of the disk reaches its surface. On the other hand if the magnetic field is strong (X-ray pulsars) the disk does not extend to the surface as magnetic pressure stops the infall. Particles are then trapped along the field lines and accretion occurs on the magnetic poles (see figure 5). Cyclotron lines in the $\mathrm{X}$-ray spectrum have brought to light the existence of fields as strong as $10^{12}$ Gauss.

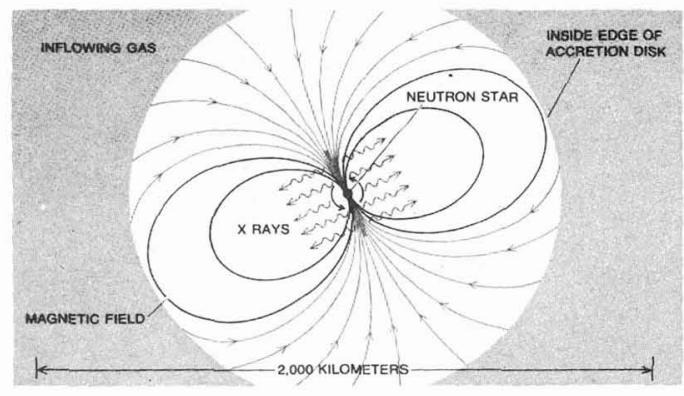

Figure 5: Special case in which the compact star in a close binary system is a neutron star with a strong magnetic field is thought to be accountable for the pulsed $X$-ray emission observed from such sources as Centaurus $\mathrm{X}-3$. In this model the star's dipole magnetic field prevents the innermost few thousand kilometers of the disk from forming. From the inside edge of the disk matter is able to continue its inward flow only along the "open" magnetic-field lines found above the star's two magnetic poles. The infalling matter enters these two "magnetic funnels," which terminate on the stellar surface in two "hot spots," each with an area of about a square kilometer. The X-ray emission is believed to arise from the two hot spots and from the turbulent gas columns above them. The rotation of the neutron star around an axis that is inclined with respect to the axis of the magnetic field causes a periodic modulation of the X-ray intensity; as a result the source appears as an X-ray pulsar.

- More massive systems belong to a second category. The only difference resides in the mass loss mechanism. Matter is ejected into space under the guise of an isotropic stellar wind, part of which is trapped by the compact companion (see figure 4a). 
In both cases, the emission occurs in a medium which is optically thick: the $\mathrm{X}$ photons undergo many Compton interactions with electrons before exiting. A typical spectrum (Cygnus $X-3$ ) is displayed on figure 6. Three components are involved to produce the continuum: A black body spectrum $(\mathrm{kT} \approx 1.2 \mathrm{keV})$, a power law which models the high energy part and a soft thermal component. In addition the source exhibits a very strong Iron line feature around $6.7 \mathrm{keV}$ made of two components: A thermal line near $6.7 \mathrm{keV}$ and fluorescence lines near $6.4 \mathrm{keV}$.

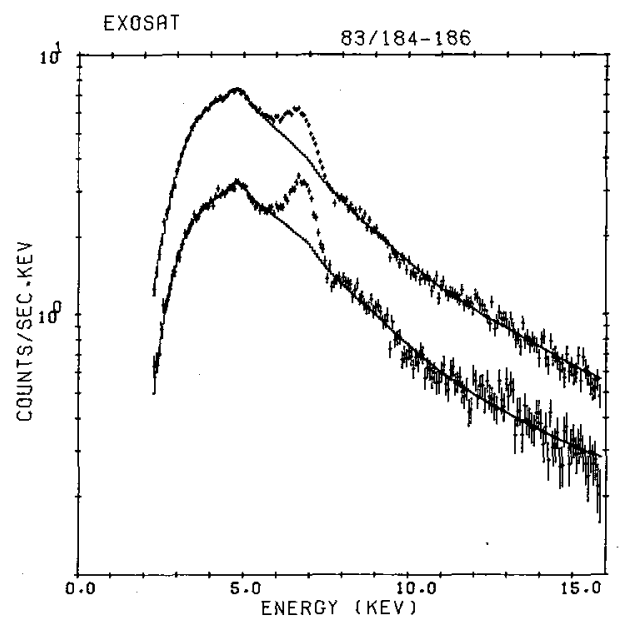

Figure 6 The $X$ ray spectrum of the binary system Cygnus $X-3$. The two curves correspond respectively to the high and low intensity states of this $4.8 \mathrm{hr}$ periodic source.

These exotic objects constitute a life-size laboratory for the physics of highly condensed matter and strong magnetic fields. Moreover they might furnish the most convincing evidence for the existence of black holes. Black holes are collapsing stars which are too massive for the neutron degeneracy pressure to stop the gravitational collapse. If most observations are consistent with the above model which involves a neutron star, in three cases the estimated mass of the compact star exceeds the equilibrium mass of a neutron star. This suggests the presence of a black hole.

We shall now consider the opposite case of an optically thin medium. 


\section{II- Supernovae remnants}

Massive stars pass through a life cycle which ends with a violent explosion known. as a Supernova. These events enrich Space with heavy elements (carbon to nickel) created in stellar nucleosynthesis. The energy released is about $10^{51}$ ergs, making of this phenomenon the main contributor to the heating of the interstellar medium. The mean time between such events in our galaxy is about 30 years, but few of them are visible to the naked eye. Kepler and Tycho were the last astronomers lucky enough to observe one. This phenomena is not peculiar to our galaxy and recently a star exploded in the nearby large magelanic cloud and is still visible at night in the southern hemisphere. It provided the first evidence of neutrino emission coming from outer space.

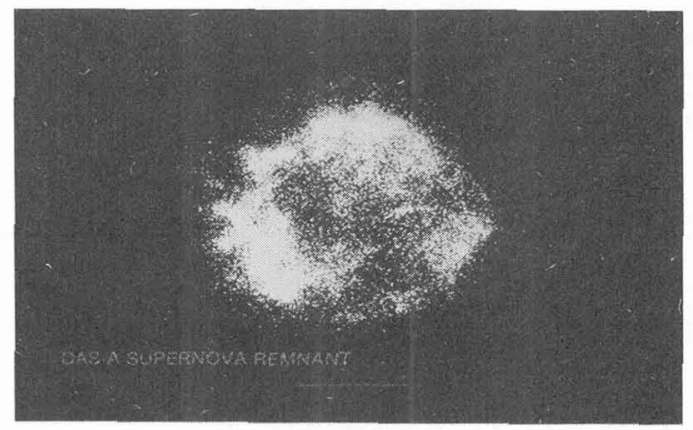

Figure 7 X-ray image of Cassiopeia A, a young supernova remnant in our galaxy. Notice the shell structure of the emission.

The X-ray emission of the remnants of Supernovae explosions (SNR) appears on images as a bright shell (see figure 7), a shape easily understood. The explosion ejects stellar matter (the ejecta) at supersonic speed into the tenuous ambient medium (about 10${ }^{2}$ particles per cubic centimeter), thus creating a outwards propagating quasi-spherical shock wave. The passage of this wave heats the interstellar matter, which reaches temperature higher than $10^{6} \mathrm{~K}$ and emits $\mathrm{X}$-rays. Besides, if the remnant is young enough the ejecta, which is not yet diluted in the surrounding medium, is heated by a secondary shock wave, which provides a supplementary source of X-ray emission. 
The spectra display heavy element lines (see figure 8), which may in principle tell us the amount and chemical composition of the ejected material. Unfortunately interpreting such data is not an easy job. Indeed the time scale for the shock propagation is smaller than the ionization characteristic time. Besides the hydrodynamical evolution itself is intricate. Thus the modelization of SNR requires to devise numerical codes which involve hydrodynamics, ionization kinetics and X-ray emission models. But in fine the accuracy of models is limited by the bad spectral resolution till now achieved. This resolution problem has been overcome in a few cases by resorting to Bragg spectrometers. However their very low efficiency (the reflectivity not exceeding $10^{-3}$ ) has till now restricted their use to very bright sources (see figure 9).

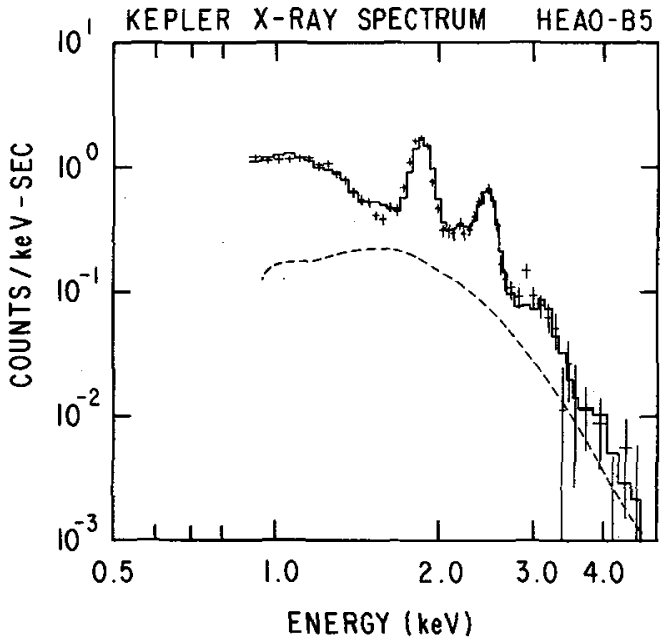

Figure 8 Spectra of the remnant of the Supernova Kepler observed. stand out clearly in this spectrum obtained by the solid state spectrometer on board the EINSTEIN satellite. (b) A proportional counter aboard EXOSAT permitted the first detection of the Iron line complex around $6.7 \mathrm{keV}$.

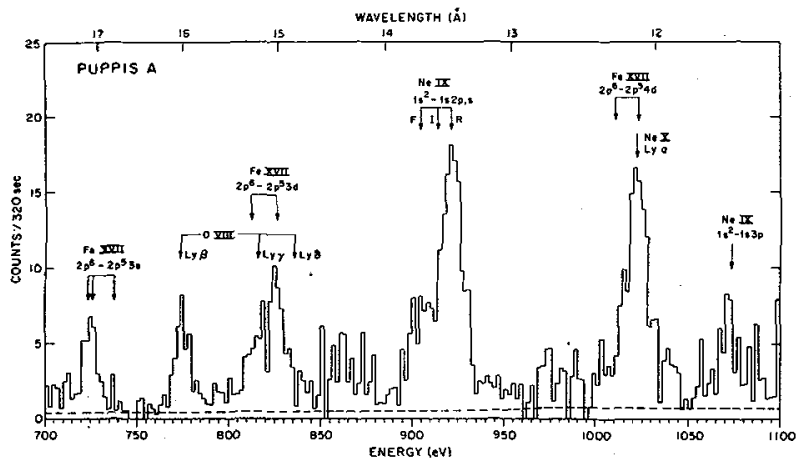

Eiqure 9 Spectrum of the old SNR Puppis A obtained by the Bragg Spectrometer on board the EINSTEIN Satellite. 
One expects, as explained in the following section, that the new generation of instruments will result in a clear progress in this field.

\section{SOME PROSPECTIVE}

The instruments under development for the future satellites XMM (European Space Agency) and AXAF (NASA) should display strongly improved capabilities in both imagery and spectroscopy:

- Charge Coupled Devices (CCD), which now equip the video camera of the man in the street, have been widely used in optical astronomy. A new generation suited for X-ray detection will combine the spectral resolution of the previous solid state spectrometers with good imaging capability (mosaics of $384 \times 576$ pixels).

- Bolometers are calorimeters which measure the energy of each incoming $X$-ray photon via the resulting increase in temperature. In principle one should gain an order of magnitude as regards the spectral resolution ( $10 \mathrm{eV}$ expected), while keeping a quantum efficiency close to the unity. However to attain this value the device must work at a temperature not exceeding $0.1 \mathrm{~K}$. This represents a real technological challenge more especially as the experiment is to be implemented on board satellites.

- Curved Bragg crystal spectrometers give access to the fine details of the $1 \mathrm{~s}-2 \mathrm{~s} \& 2 \mathrm{p}$ line complexes for He and H-like ions of the most abundant elements in Space (such as Oxygen, Silicon, Sulfur and Iron). A resolution $\lambda / \Delta \lambda \approx 1500$ is expected for the observation of Iron lines around $1.85 \AA$.

Besides new type of mirrors with much larger collecting areas (in addition to a better resolution) should make up for the relatively low sensibility of Bragg spectrometers. Moreover they will extend our horizon by a factor of 10 , thus enabling us to detect extremely remote objects, such as quasars.

Astronomers think this brand new generation of instruments will help them to solve intriguing problems such as:

- What remains when a star explodes?

- Do black holes really exist?

- How did galaxy clusters form and evolve?

- What is the true nature of Quasars? 


\section{FURTHER READING}

For more information, the reader may consult:

[1] X-Ray Astronomy with the EINSTEIN Satellite, R. Giacconi and G. Setti Editors, Reidel, Dordrecht, 1981.

[2] X-Ray Astronomy, Proceedings of the XV ESLAB Symposium, R.D. Andresen Editor, Space Science Reviews, 30, 1-4, Reidel, Dordrecht, 1981.

[3] Supernova Remnants and their X-Ray Emission, J. Dantziger and P. Gorenstein Editors, International Astronomical Union Symposium $n^{\circ} 101$, Reidel, Dordrecht, 1983.

[4] X-Ray Astronomy 84, International Symposium on X-Ray Astronomy (Bologna,June 1984), M. Oda and R. Giacconi Editors, Institute of Space and Astronautical Science, Tokyo, 1984.

[5] X-Ray Astronomy in the EXOSAT era, Proceedings of the XVIII ESLAB Symposium, A. Peacock Editor, Space Science Reviews, 40, n¹-4, 1985.

\section{SOURCES OF FIGURES}

Figure 2 a: $\quad$ R. Giacconi, 1980, Scientific American, 242, number 2, 70.

Figure 2 b: $\quad$ 'The European X-Ray Astronomy Satellite Exosat observers guide', partll, page 9, ESA publication.

Figure 3 a: $\quad$ M. Bitter et al., 1979, Physical Review Letters, 43, 129.

Figure 3 b: $\quad$ R. Mitchell and R. Mushotzky, 1980, Astrophysical Journal, 236, 730.

Figures 4 \& 5: H. Gursky and E. Van den Heuvel,1975, Scientific American, 232, number 3, 24.

Figure 6: $\quad$ M. Van der Klis et al., 1985, Space Science Reviews, 40, 297.

Figure 7: $\quad$ S. Murray et al., 1980, Astrophysical Journal Letters, 234, L69.

Figure 8 a: $\quad$ R.H. Becker et al., 1980, Astrophysical Journal Letters, 237, L77.

b: J. Ballet et al., Colloquium 'Supernovae remnants and their interaction with the interstellar medium', Pentington, Canada, June 1987.

Figure 9: $\quad$ P.F. Winkler et al., 1981, Astrophysical Journal Letters, 246, L27. 